\title{
Antigenic outer membrane proteins prediction of Pasteurella multocida serotype $\mathrm{B}: 2$
}

\author{
Farahani Muhammad Azama, , Mohd. Zamri-Saad ${ }^{\mathrm{a}}$, Raha Abdul Rahimª, Pramote Chumnanpoen ${ }^{\mathrm{d}}$, \\ Teerasak E-kobon ${ }^{\mathrm{b}}$, Sarah Othman ${ }^{\mathrm{a}, \mathrm{e}^{*}}$, \\ aDepartment of Cell and Molecular Biology, Faculty of Biotechnology and Biomolecular Sciences, Universiti Putra Malaysia, 43400 UPM \\ Serdang, Selangor, Malaysia \\ ${ }^{b}$ Department of Genetics, Faculty of Science, Kasetsart University, Ladyao, Chatuchak, 10900 Bangkok, Thailand \\ ${ }^{'}$ Research Centre for Ruminant Diseases, Faculty of Veterinary Medicine, Universiti Putra Malaysia, 43400 UPM, Serdang, Selangor, \\ Malaysia \\ ${ }^{d}$ Department of Zoology, Faculty of Science, Kasetsart University, Ladyao, Chatuchak, 10900 Bangkok, Thailand \\ ${ }^{e} U P M-M A K N$ A Cancer Research Laboratory, Institute of Bioscience, Universiti Putra Malaysia, 43400 UPM, Serdang, Selangor, Malaysia
}

Received 8th July 2020 / Accepted 9th November 2020

\begin{abstract}
Outer membrane proteins (OMPs) are one of the prominent virulence factor or immunogenic element of Pasteurella multocida which are responsible for eliciting immune responses in multiple infected hosts. Identification of these proteins allows researchers to target OMPs to be manipulated as a vaccine against bacterial infection. Precise and rapid bioinformatics tools allow researchers to perform in silico analysis to extract putative OMPs from the genome information. In this study, we have successfully identified 105 putative OMPs of P. multocida subsp. multocida strain PMTB2.1 through computational prediction tools including a subcellular localisation predictor, PSORTb v3.0 followed by a lipoprotein predictor, LipoP 1.0 and a $\beta$-barrel transmembrane protein predictor, BOMP for sub-classification of the OMPs into 53 integral and 52 peripheral OMPs of this strain. The manipulation of antigenic epitope predictors and the antigenicity score filtering identified nine putative antigenic OMPs. These putative predicted antigenic OMPs of this pathogen will provide crucial initial guidance for the experimental identification and selection of antigenic protein(s) for the development of future haemorrhagic septicaemia (HS) vaccine.
\end{abstract}

Keywords: antigenic outer membrane proteins, OMPs, Pasteurella multocida, outer membrane protein prediction, haemorrhagic septicaemia

\section{INTRODUCTION}

Haemorrhagic septicaemia (HS) is a chronic and fatal disease of domesticated ruminant animals especially buffaloes and cattle. In Asia, the responsible pathogen of this disease is Pasteurella multocida serotype B:2. Vaccination is a mean of prevention as there is no treatment after the occurrence of disease. To date, oil-adjuvant and whole-killed vaccines are routinely used for vaccination (Alwis, 1999; Benkirane \& De Alwis, 2002; Zamri-Saad, 2013). Even though these vaccines enabled protection towards the animals post-vaccination, there are several drawbacks on the vaccine's administration and its effectiveness (Zamri-Saad, 2013). Currently, there are widely studied live attenuated vaccine and also subunit vaccine derived from the pathogen, such as major

*Author for correspondence: Sarah Othman, Department of Cell and Molecular Biology, Faculty of Biotechnology and Biomolecular Sciences, Universiti Putra Malaysia, 43400 UPM Serdang, Selangor, Malaysia. Email sarahothman@upm.edu.my / sarahothma07@gmail.com 
outer membrane protein $\mathrm{H}(\mathrm{OmpH})$ and Pasteurella lipoprotein P (PlpP); developed to overcome the disadvantages of conventional vaccines (Tabatabaei et al., 2002; Lee et al., 2007; Wu et al., 2007).

Outer membrane proteins (OMPs) are known as one of the virulence factors and are strong antigenic agents of P. multocida (Basagoudanavar et al., 2006). The OMPs of Gram-negative bacteria and also $P$. multocida are divided into two major components which are the integral membrane proteins (intrinsic proteins) and the peripheral membrane proteins (extrinsic proteins) in which the prior are embedded within the lipid bilayer, but the latter are only attached to the outer of the lipid bilayer. Peripheral proteins are commonly anchored to the membrane using a covalently linked-lipid anchor that enters the membrane bilayer (Lodish et al., 2007). Bioinformatics analysis or prediction towards proteins localisation in bacteria is crucial in order to comprehend their structure and function, particularly in the disease development by the pathogen. Efforts towards the identification of putative OMPs of P. multocida were seen through in silico analysis to select potential vaccine targets for other strains such as avian (Al-Hasani et al., 2007; E-komon et al., 2012; Hatfaludi et al., 2012) and porcine strains (E-komon et al., 2012) of $P$. multocida.

There are several OMPs targeted vaccines that have been developed. Previous study reported that OMPs fraction of P. multocida serotype B:2 was able to protect buffalo and calves against post-challenged (Pati et al., 1996). They showed an effort to immunize mice against virulent $P$. multocida challenge using purified OMPs mixture with $67 \%$ protection rate as compared to the commercial whole-killed vaccine protection at the rate of $84 \%$ (Srivastava, 1998). Similar to Basagoudanavar et al. (2006), Joshi et al. (2013) reported that $100 \%$ protection was provided by the OMPs vaccine when compared to the commercial vaccine which only enabled protection up to $83.33 \%$. This report stated that OMPs fraction are potent antigens suitable to be manipulated as vaccine against $P$. multocida $\mathrm{B}: 2$ infection. Some individual OMPs were thoroughly studied in their role of immunity and developed as vaccine candidate against pasteurellosis. It was reported that purified recombinant vaccine of lipoprotein $\mathrm{E}$ (PlpE) enabled $63-100 \%$ protection following wild-type challenge in chickens (Wu et al., 2007). Besides that, two OM polypeptides, Fur and $\mathrm{OmpH}$ were targeted for attenuation, and the fur ompH mutant enabled total protection towards the challenged mice. Mutations towards the proteins were found to improve the protection capacity of the mutant strain in the infected mice (Garrido et al., 2008). Identification of unique OMPs from $P$. multocida serotype B:2 was reported in an elaborate proteomic study by Prasannavadhana et al. (2014) which also included several key iron-regulating proteins. Application of MALDI-TOF mass spectrometry analysis has identified 22 OMPs from the OMP profile of an Indian vaccine strain P. multocida B:2 P52. Another study on P. multocida serotype $B$ isolated in China has revealed promising cross-protective vaccine candidates against the infection of bovine $P$. multocida serotype A and B in the mouse model (Du et al., 2017). From the challenge analysis, two vaccine candidates manipulated from a transmembrane protein and an $\mathrm{ABC}$ transporter binding protein respectively showed protection when challenged (Du et al., 2017).

Identification of OMPs to be manipulated as protective antigens are crucial for the potential vaccine development process. Several easy-to-use and precise bioinformatics tools are currently available to aid researchers in identifying the candidate proteins or genes related to the antigenic components of a pathogen. This approach is known as reverse vaccinology that offers time-saving and problem-solving approach against conventional vaccinology design (Rappuoli, 2001). Through this approach, notable vaccines were found particularly for human infections which are group B Streptococcus (GBS) infection and Chlamydia infection (Maione et al., 2005; Thorpe et al., 2007). Currently, there are no recent researches on the identification of $P$. multocida OMP antigenic profiles in bovine HS in Southeast Asian countries, especially Malaysia. Hence, this study was aimed to identify the putative antigenic OMPs of local strain $P$. multocida B:2 subsp. multocida PMTB2.1 from its available genome as potential candidates for further vaccine development towards HS by using bioinformatics prediction tools for subcellular localisation, OMPs sub-classification, antigenicity 
determination and selection of the putative antigenic OMPs.

\section{MATERIALS AND METHODS}

\section{Pasteurella multocida proteome \\ Pasteurella multocida subsp. multocida PMTB2.1 is a pathogenic P. multocida B:2 isolated from an HS outbreak in Malaysia, and the genome has been sequenced and deposited in the NCBI database (Acc. No. PRJNA236642). The sequences of Pasteurella multocida subsp. multocida PMTB2.1 were retrieved from the NCBI database (https://www.ncbi.nlm.nih.gov).}

\section{Identification of outer membrane proteins}

Subcellular protein localisation prediction was performed on the listed whole proteome of Pasteurella multocida subsp. multocida PMTB2.1 to identify proteins located in the outer membrane region of $P$. multocida known as the outer membrane proteins (OMPs) by using PSORTb v3.0 program (https://www.psort.org/psortb/). PSORTb is the most precise and straightforward subcellular protein localiser that is also used for protein function annotation, identification of cell surface or secreted drug targets and identification of microbes biomarkers. The developers of PSORTb v3.0 has stated a significant increase in protein coverage, especially towards the precision and recalled identified protein compared to its previous version, PSORTb v2.0 (https://www.psort.org/psortb2/index.html) (Yu et al., 2010). The algorithm of PSORTb program enabled assignation of the proteins in the bacteria based on the sequences of the amino acids (Yu et al., 2010). The proteins were submitted to the PSORTb server in FASTA format. The input was identified using ten prediction modules. The output that classified into OMPMotif and OMSVM modules were considered as OMPs. Proteins with a final prediction score of more than eight were chosen for subsequent sub-classification of the predicted OMPs.

Lipoproteins are peripheral membrane proteins that interact with the lipid bilayer through the fatty acid chain (Lodish et al., 2007). LipoP v1.0 program
(http://www.cbs.dtu.dk/services/LipoP/) is a highly sensitive and specific tool that enabled the determination of lipoproteins from the whole proteome (Juncker \& Willenbrock 2003). LipoP was designed based on the Hidden Markov's model (HMM) to predict SPaseII-cleaved lipoproteins between SPaseI-cleaved, cytoplasmic and transmembrane proteins with $96.8 \%$ accuracy (Juncker \& Willenbrock 2003). Lipoproteins have a conserved c-region which is known as lipobox. This region functions as the recognition site for the cleavage of SPaseII (Juncker et al., 2003). LipoP was used to support PSORTb analysis due to the disadvantage in detecting peripheral membrane proteins of the latter. FASTA format of the listed whole proteomes was used as input for LipoP and the output can be returned in extensive graphics or simple text. The proteins scored as signal peptidase II (SpaseII) were regarded as putative outer membrane lipoprotein.

The whole proteome was further analysed to determine the presence of $\beta$-barrel OMPs (Berven et al., 2004). BOMP predictor (http://services.cbu.uib.no/tools/bomp) is a reliable and fast tool to enable identification of $\beta$ barrel integral proteins and has been reported to have $80 \%$ accuracy and $88 \%$ recall (Berven et al., 2004). The protein sequences were submitted to BOMP program using FASTA format as input. The determined $\beta$-barrel OMPs was showed in total counted $\beta$-barrel OMPs and tabulated based on the category of prediction reliability. The tabulated output was assigned into five categories named as category 1 to 5 where 5 is the most probable $\beta$-barrel OMPs.

The predictive identification of putative OMPs was performed as described in Figure 1A. Predicted putative OMPs were confirmed by reviewing their subcellular information in the UniProt protein database (https://www.uniprot.org/) to identify possible false positive proteins.

\section{Prediction of outer membrane proteins' antigenicity}

The predicted putative OMPs were used as input in FASTA format for the antigenicity prediction using two programs, MULTIPRED2 (http://projects.met-hilab.org/multipred2/) and VaxiJen2.0 (http://www.ddgpharmfac.net/vaxijen/VaxiJen/VaxiJen.html) 
(Figure 1A). Both programs apply different approaches in determining protein antigenicity. MULTIPRED2 prediction is based on the protein sequence complementation towards multiple alleles of the human leukocyte antigen (HLA). The program was used to predict the promiscuous binders of the OMPs that can bind to the HLA class II supertypes (Zhang et al., 2011). Majority of MHC class II epitope binding algorithm used prediction towards human leukocyte antigens (HLA) as compared to other leukocyte antigens particularly bovine leukocyte antigens (BoLA). However, it was found that there was a certain pseudo-sequence (representation of the MHC-II complex polymorphic binding pockets) similarity between both species' alleles to approximate the protein binding in bovine (Farrell et al., 2016). Hence, the application of the selected HLA alleles that were found shown to be similar to BoLA by Farrel et al. (2016). We predicted antigens that would bind specifically towards bovine BoLA. The input OMPs were submitted in FASTA format to MULTIPRED2 tool with default peptide length of nine. The prediction was achieved by detecting non-binders or binders to at least $50 \%$ of the alleles supertypes that indicated the presence of strong promiscuous binders.

VaxiJen2.0 is independent towards sequence alignment. Instead, it considered the protein properties and neighbour effects by incorporating three $z$ descriptors (hydrophobicity, steric properties and polarity) towards auto-cross covariance (ACC) pre-processing of proteins physiochemical properties, and hence, assigning the proteins into probable or non-probable antigens. Therefore, identification of the antigens was efficiently aided with the accounts of intermolecular interactions combination (Doytchinova \& Flower 2007; Zaharieva et al. 2017). The targeted proteins in VaxiJen 2.0 were predicted using the ACC method with a threshold of 0.5 in which the proteins with a score of more than 0.5 are determined as antigenic.

All putative antigenic OMPs were subsequently analysed using Protein Basic Local Alignment Search Tool (BLASTP) (https://blast.ncbi.nlm.nih.gov/) to support their predicted antigenicity by identifying orthologues in closely-related bacterial species with experimental antigenic evidence and further annotation of the unknown or hypothetical OMPs. The proteins were scored from the nonredundant protein sequences database using BLOSUM62 matrix with an expected threshold of 10. The identified putative OMPs with E-value closest to zero and with $\geq 50 \%$ identity and $\geq 80 \%$ sequence coverage was selected and ranked by the VaxiJen antigenicity score. The proteins with score above 0.7 were considered as potential antigens for the vaccine design. The literature search was also used to add on the information of the determined putative OMPs. Molecular weight (MW) and isoelectric point (pI) of all the putative antigenic OMPs were determined using the Compute $\mathrm{pI} / \mathrm{Mw}$ tool by ExPASy. Antigenic sites of each protein were determined by using EMBOSS 'Antigenic' tool with antigenic region length of 6 (Baliga \& Venugopal 2018).

\section{RESULTS}

\section{Localisation of outer membrane proteins}

Three individual bioinformatics prediction tools were used to determine and to categorise total OMPs from the whole proteome of Pasteurella multocida subsp. multocida PMTB2.1. A total of 105 putative OMPs were successfully predicted in this study (Figure 1B). The subcellular localisation predictor, PSORTb, was able to localise 53 OMPs from the whole proteome. Subsequently, 52 lipidified OMPs or OM lipoproteins from a total of 105 putative OMPs were recognized in the HS pathogen from the lipoprotein prediction analysis. Out of the 52 OM lipoproteins, 13 proteins were recognized by both PSORTb and LipoP predictors as peripheral OMPs. BOMP predictor tool enabled the identification of $39 \beta$-barrel folded OMPs that overlapped with 26 of the proteins predicted by PSORTb. There are no overlapping proteins by LipoP and BOMP observed. Hence, confirming the specificity of LipoP to detect peripheral OMPs (lipoproteins) and BOMP to identify integral OMPs $(\beta$-barrel folded OMPs) (Juncker \& Willenbrock, 2003; Berven et al., 2004). The calculated false positive of the OMPs determination was $4.76 \%$. 

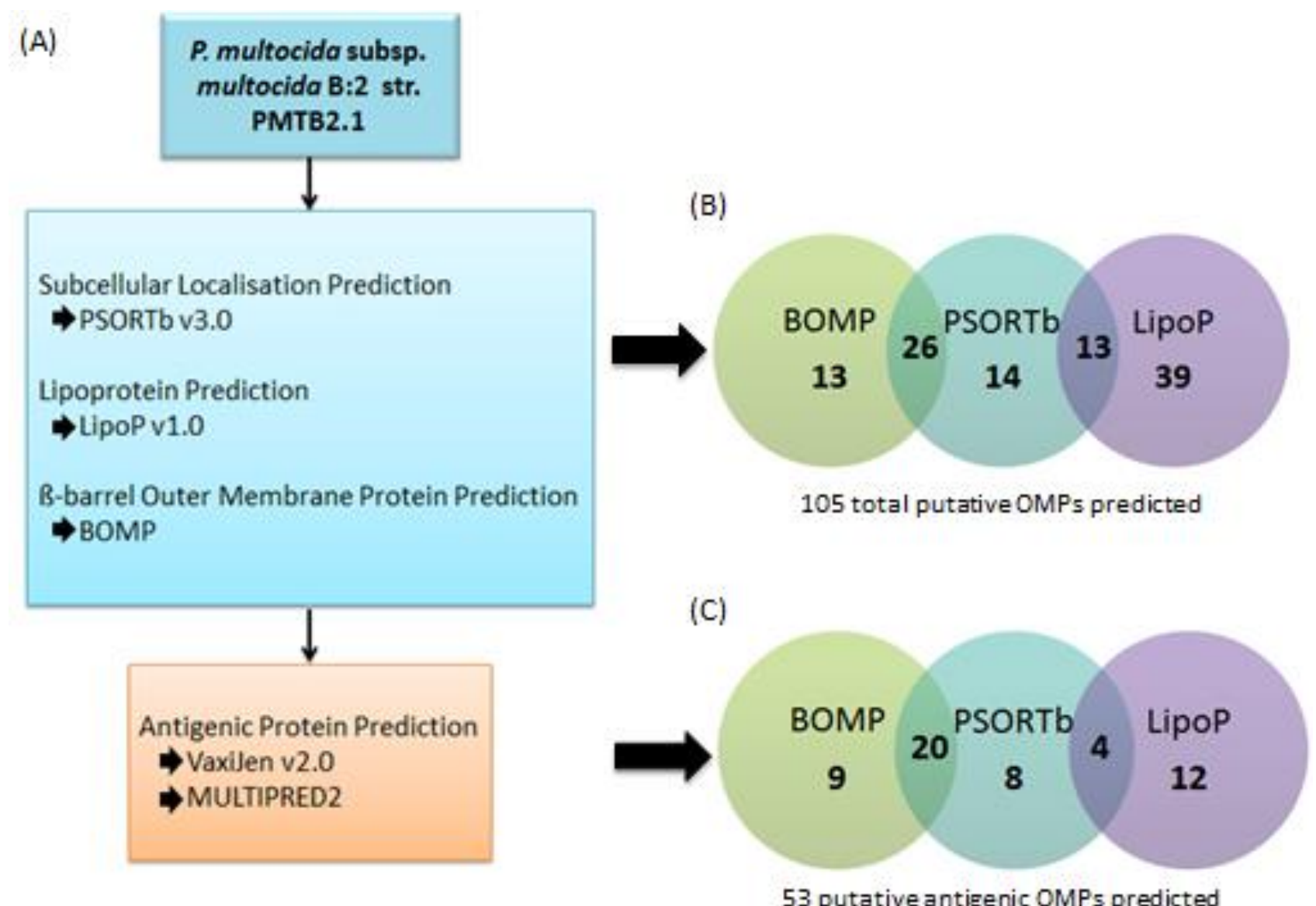

Figure 1. (A) Overview of outer membrane proteins (OMPs) prediction. The identification of putative OMPs from the whole proteome of P. multocida subsp. multocida PMTB2.1 was performed concurrently. Listed identified OMPs were further categorised into probable antigen and non-antigen. (B) Bioinformatics prediction of outer membrane proteins from P. multocida subsp. multocida PMTB2.1 using PSORTb (53 OMPs), LipoP (52 OMPs) and BOMP (39 OMPs). The total predicted OMPs are 105 putative proteins. (C) Bioinformatics prediction of outer membrane proteins from P. multocida subsp. multocida PMTB2.1 using MULTIPRED2 and VAxiJen. The antigenic OMPs predicted by both predictors are 53 putative proteins out of 105 total putative OMPs.

\section{Antigenic outer membrane proteins}

Putative antigenic OMPs were identified using two individual predictors, MULTIPRED2 and Vaxijen (Figure 1C). The programs have different approaches to determine the protein antigenicity given that MULTIPRED2 is a sequencedependent programme whereas VaxiJen is a sequence-independent programs (Doytchinova \& Flower 2007; Zhang et al. 2011). Listed proteins from the OMPs identification were used as input for antigenicity determination whereby the proteins identified by both MULTIPRED2 and VaxiJen were accepted as antigenic. Majority of the antigenic proteins ( 32 proteins) were identified from PSORTb whereas 29 antigenic OMPs determined from BOMP and 16 antigenic
OMPs determined from LipoP. A total of 53 putative antigenic OMPs were identified and listed in Table 1 with respective detailed length, molecular weight, pI value and the number of antigenic sites. The number of antigenic sites most likely corresponded to the length of the amino acid aside from identified hydrophobic antigenic residues (Kolaskar et al., 1990). Hence, the antigenic sites identified from AMM82057.1 and AMM82054.1 were the highest among other proteins. Table 1 has also described the information of all the antigenic OMPs identified in this study with other relevant protection experiments. However, information retrieved from the protection studies of the proteins from $P$. multocida B:2 was very scarce unlike other 
serotypes of $P$. multocida such as serotype A. The information relevant to the organism corresponding to the protein of interest, the protection type (homologous or heterologous protection) and the vaccine types were shown in Table 1. From this study, we narrowed down the predicted putative antigenic OMPs of $P$. multocida $\mathrm{B}: 2$ into nine OMPs from 53 predicted antigenic OMPs as vaccine targets for future HS vaccine development; OmpH, OmpA, OmpW, OmpV, Opa, hypothetical proteins AMM82273.1 and AMM82618.1, TamB, and YopT (AMM82057.1). These putative antigenic OMPs were selected based on the antigenicity score in which scores above than the threshold of 0.7 is regarded as highly antigenic OMPs.

\section{DISCUSSION}

Bioinformatics analysis of proteins is critical especially towards identifying putative antigens to be targeted for vaccine development. There are numerous tools currently that are fast, precise and user-friendly especially for users with a minimal bioinformatics research background. In this study, these three tools were used to predict each specified category of the outer membrane proteins which was performed by identifying subcellular localisation, prediction of outer membrane lipoproteins and $\beta$-barrel integral OMPs.

The respective bioinformatics tools applied in this study was also observed for OMPs determination in various pathogenic bacteria. The precision of the PSORTb v3 was seen to be improved from its previous version and other subcellular localiser tools with the significant increase in recall rate of $94.1 \%$ and precision rate of $97.3 \%$ (Yu et al., 2010). OMPs from Bordetella pertussis, Salmonella typhii, Vibrio harveyi and Corynebacterium psendotuberculosis were characterised through the application of PSORTb tool to comprehend on their pathogenicity and to target the determined proteins for future vaccine improvement (Al-Hasani et al., 2007; Bottero et al., 2012; E-komon et al., 2012; Ferrer-Navarro et al., 2016; Mohd Aris et al., 2016; Santana-Jorge et al., 2016). The precision and recall rate of BOMP was satisfactory with a score of $80 \%$ precision and
88\% recall (Berven et al., 2004). Furthermore, BOMP was applied for the determination of $\beta$ barrel OMPs in P. multocida and Veillorella parvula (Pandey et al., 2016; Cao et al., 2017; Poppleton et al., 2017). Manipulation of LipoP tool for the identification of lipoproteins was observed with 96.8\% accuracy (Juncker \& Willenbrock 2003). The lipoprotein prediction was also used previously for vaccine candidate selection; 36 lipoproteins were identified from Francisella noatunesis (Shahin et al., 2018) and two antigenic lipoproteins were targeted as vaccine candidate from Vibrio anguillarum (Baliga et al., 2018).

In a previous study, analysis towards an avian strain and porcine strain sequences revealed 98 and 107 putative OMPs respectively (E-komon $e t$ al., 2012). However, Al-Hasani et al. (2007) have predicted 129 proteins with the same avian strain, P. multocida Pm70. About $18.09 \%$ of OMPs were also identified as in E-komon et al. (2012) study, and $21.13 \%$ of the OMPs were similar to Hatfaludi et al. (2012) study, hence confirming that the proteins predicted in this study is indeed proteins that localise in the outer membrane. The false positive of this study was revealed as $4.97 \%$ indicating our true OMPs with 95.24\%. Therefore, of more than $70 \%$ of the identified putative OMPs of this study is strongly believed from the contribution of novel or unique proteins presence from different strains used in respective studies. Numerous hypothetical or unknown proteins detected in both studies and also in this study were unable to be scrutinized further due to information limitation, thus, these proteins are also the novel putative OMPs identified in respective strains. Furthermore, various proteins identified in this study were also acknowledged as OMPs by previous findings in P. multocida. Both of porins, OmpA and $\mathrm{OmpH}$ were reported as OMPs in avian and porcine strains of $P$. multocida. Porin, OmpW and acetylglucosamine transferase, Opa were only found in avian $P$. multocida. Meanwhile, an iron-limiting protein, $\mathrm{HgbA}$, and an efflux channel protein, TolC were reported as OMPs in both P. multocida strains (E-komon et al., 2012; Hatfaludi et al., 2012). 
Table 1. List of the identified putative antigenic OMPs of P. multocida subsp. multocida PMTB2.1.

\begin{tabular}{|c|c|c|c|c|c|c|c|c|c|c|}
\hline $\begin{array}{l}\text { Protein } \\
\text { functional } \\
\text { groups }\end{array}$ & $\begin{array}{l}\text { Accession } \\
\text { No. }\end{array}$ & Description & $\begin{array}{l}\text { Length } \\
\text { (aa) }\end{array}$ & $\begin{array}{l}\text { MW } \\
(\mathrm{kDa})\end{array}$ & $\begin{array}{l}\text { Theoret } \\
\text { ical pI }\end{array}$ & $\begin{array}{l}\text { No. } \\
\text { of } \\
\text { antig } \\
\text { enic } \\
\text { sites }\end{array}$ & $\begin{array}{l}\text { Associating } \\
\text { organism }\end{array}$ & $\begin{array}{l}\text { Protection } \\
\text { type }\end{array}$ & Reference & Vaccine type \\
\hline \multirow[t]{12}{*}{ Porins } & \multirow[t]{2}{*}{$\begin{array}{l}\text { AMM81683. } \\
1\end{array}$} & \multirow[t]{2}{*}{ Porin, OmpA } & \multirow[t]{2}{*}{353} & \multirow[t]{2}{*}{37.95} & \multirow[t]{2}{*}{9.01} & \multirow[t]{2}{*}{16} & $\begin{array}{l}P . \quad \text { multocida } \\
\text { serotype A:3 }\end{array}$ & No protection & \multirow{4}{*}{$\begin{array}{l}\text { (Gatto et al., } \\
\text { 2002; Dabo et } \\
\text { al., 2008) } \\
\text { (Hatfaludi et } \\
\text { al., 2012) } \\
\text { (Luo et al., } \\
\text { 1999) } \\
\text { (Hatfaludi et } \\
\text { al., 2012) }\end{array}$} & Recombinant \\
\hline & & & & & & & $\begin{array}{l}P . \quad \text { multocida } \\
\text { serotype A }\end{array}$ & No protection & & Recombinant \\
\hline & \multirow{2}{*}{$\begin{array}{l}\text { AMM81212. } \\
1\end{array}$} & \multirow{2}{*}{$\begin{array}{l}\text { Major outer membrane } \\
\text { protein, OmpH }\end{array}$} & \multirow[t]{2}{*}{353} & \multirow[t]{2}{*}{38.54} & \multirow[t]{2}{*}{7.72} & \multirow[t]{2}{*}{15} & P. multocida X-73 & Homologous & & Recombinant \\
\hline & & & & & & & $\begin{array}{l}P . \quad \text { multocida } \\
\text { serotype A }\end{array}$ & No protection & & Recombinant \\
\hline & \multirow[t]{2}{*}{$\begin{array}{l}\text { AMM81213. } \\
1\end{array}$} & \multirow[t]{2}{*}{ Porin, $\mathrm{OmpH} 2$} & \multirow[t]{2}{*}{350} & \multirow[t]{2}{*}{38.82} & \multirow[t]{2}{*}{8.84} & \multirow[t]{2}{*}{15} & $\begin{array}{l}P . \quad \text { multocida } \\
\text { serotype A }\end{array}$ & Heterologous & $\begin{array}{l}\text { (Garrido et } \\
\text { al., 2008) }\end{array}$ & $\begin{array}{l}\text { Inactivated } \\
\text { bacterin }\end{array}$ \\
\hline & & & & & & & $\begin{array}{l}\text { P. multocida } \\
\text { serotype A }\end{array}$ & Homologous & $\begin{array}{l}\text { (Gong et al., } \\
\text { 2013) }\end{array}$ & DNA \\
\hline & \multirow[t]{2}{*}{$\begin{array}{l}\text { AMM81158. } \\
1\end{array}$} & \multirow[t]{2}{*}{$\begin{array}{l}\text { Outer membrane protein } \\
\mathrm{W}, \text { OmpW }\end{array}$} & \multirow[t]{2}{*}{204} & \multirow[t]{2}{*}{21.88} & \multirow[t]{2}{*}{9.16} & \multirow[t]{2}{*}{9} & $\begin{array}{l}P . \quad \text { multocida } \\
\text { serotype A }\end{array}$ & No protection & $\begin{array}{l}\text { (Hatfaludi et } \\
\text { al., 2012) }\end{array}$ & Recombinant \\
\hline & & & & & & & E. coli $\mathrm{K} 12$ & Homologous & $\begin{array}{l}\text { (Wu et al., } \\
2013)\end{array}$ & $\begin{array}{l}\text { Live } \\
\text { attenuated }\end{array}$ \\
\hline & \multirow[t]{2}{*}{$\begin{array}{l}\text { AMM81732. } \\
1\end{array}$} & \multirow[t]{2}{*}{ Porin, OmpC } & \multirow[t]{2}{*}{372} & \multirow[t]{2}{*}{41.46} & \multirow[t]{2}{*}{8.89} & \multirow[t]{2}{*}{12} & \multicolumn{2}{|l|}{ S. flexneri } & $\begin{array}{l}\text { (Bernardini et } \\
\text { al., 1993) }\end{array}$ & \multirow[t]{2}{*}{$\begin{array}{l}\text { Live } \\
\text { attenuated }\end{array}$} \\
\hline & & & & & & & E. coli & & $\begin{array}{l}\text { (Liu et al., } \\
\text { 2012) }\end{array}$ & \\
\hline & $\begin{array}{l}\text { AMM81037. } \\
1\end{array}$ & $\begin{array}{l}\text { Outer membrane protein } \\
\text { V, OmpV }\end{array}$ & 257 & 28.66 & 9.65 & 10 & $\begin{array}{l}V . \\
\text { parabaemolyticus }\end{array}$ & Homologous & $\begin{array}{l}\text { (Mao et al., } \\
2007 \text { ) }\end{array}$ & Recombinant \\
\hline & $\begin{array}{l}\text { AMM80966. } \\
1\end{array}$ & $\begin{array}{l}\text { Porin, outer membrane } \\
\text { protein P1 }\end{array}$ & 424 & 45.77 & 8.80 & 15 & $\begin{array}{l}\text { Non-typeable } \\
\text { H. influenzae }\end{array}$ & Heterologous & $\begin{array}{l}\text { (Bolduc et al., } \\
\text { 2000) }\end{array}$ & Recombinant \\
\hline IROMPs & $\begin{array}{l}\text { AMM82688. } \\
1\end{array}$ & TonB-dependent receptor & 668 & 76.28 & 9.10 & 25 & & & & \\
\hline & $\begin{array}{l}\text { AMM82668. } \\
1\end{array}$ & TonB-dependent receptor & 778 & 88.10 & 8.92 & 35 & & & & \\
\hline & $\begin{array}{l}\text { AMM82428. } \\
1\end{array}$ & TonB-dependent receptor & 805 & 90.96 & 8.98 & 37 & & & & \\
\hline & $\begin{array}{l}\text { AMM82074. } \\
1\end{array}$ & TonB-dependent receptor & 727 & 81.39 & 9.44 & 25 & & & & \\
\hline
\end{tabular}




\begin{tabular}{|c|c|c|c|c|}
\hline $\begin{array}{l}\text { AMM81704. } \\
1\end{array}$ & TonB-dependent receptor & 811 & 93.14 & 8.81 \\
\hline $\begin{array}{l}\text { AMM81639. } \\
1\end{array}$ & TonB-dependent receptor & 922 & 104.83 & 7.80 \\
\hline $\begin{array}{l}\text { AMM81164. } \\
1\end{array}$ & TonB-dependent receptor & 996 & 113.48 & 8.79 \\
\hline $\begin{array}{l}\text { AMM81163. } \\
1\end{array}$ & TonB-dependent receptor & 992 & 114.36 & 9.02 \\
\hline $\begin{array}{l}\text { AMM80956. } \\
1\end{array}$ & TonB-dependent receptor & 809 & 90.97 & 8.87 \\
\hline $\begin{array}{l}\text { AMM81635. } \\
1\end{array}$ & $\begin{array}{l}\text { TonB-dependent } \\
\text { hemoglobin/ transferrin/ } \\
\text { lactoferrin family receptor }\end{array}$ & 784 & 89.56 & 9.19 \\
\hline $\begin{array}{l}\text { AMM81129. } \\
1\end{array}$ & $\begin{array}{ll}\text { Hemoglobin } & \text { binding } \\
\text { protein } \mathrm{A}, \mathrm{HgbA} & \end{array}$ & 971 & 110.37 & 8.77 \\
\hline
\end{tabular}

\begin{tabular}{|c|c|c|c|}
\hline & $\begin{array}{l}\text { AMM82687. } \\
1\end{array}$ & $\begin{array}{l}\text { Outer membrane } \\
\text { autotransporter barrel } \\
\text { protein }\end{array}$ & 1248 \\
\hline \multirow{9}{*}{$\begin{array}{l}\text { Membrane } \\
\text { assembly- } \\
\text { associated } \\
\text { proteins }\end{array}$} & $\begin{array}{l}\text { AMM82596. } \\
1\end{array}$ & $\begin{array}{l}\text { LPS assembly protein, } \\
\text { LptD }\end{array}$ & 782 \\
\hline & $\begin{array}{l}\text { AMM82508. } \\
1\end{array}$ & Peptidase family m 23 & 423 \\
\hline & $\begin{array}{l}\text { AMM81068. } \\
1\end{array}$ & $\begin{array}{l}\text { Peptidoglycan-associated } \\
\text { lipoprotein, } \mathrm{Pal}^{*}\end{array}$ & 150 \\
\hline & $\begin{array}{l}\text { AMM81019. } \\
1\end{array}$ & $\begin{array}{l}\text { Polysaccharide } \\
\text { protein, Wza* }\end{array}$ & 387 \\
\hline & $\begin{array}{l}\text { AMM82133. } \\
1\end{array}$ & $\begin{array}{l}\text { Outer membrane protein } \\
\text { assembly factor, BamA }\end{array}$ & 791 \\
\hline & $\begin{array}{l}\text { AMM80985. } \\
1\end{array}$ & $\begin{array}{l}\text { Outer membrane protein } \\
\text { assembly factor, BamC* }\end{array}$ & 337 \\
\hline & $\begin{array}{l}\text { AMM81444. } \\
1\end{array}$ & $\begin{array}{l}\text { Outer membrane protein } \\
\text { assembly factor, BamE* }\end{array}$ & 272 \\
\hline & $\begin{array}{l}\text { AMM82722. } \\
1\end{array}$ & $\begin{array}{l}\text { Thiamine biosynthesis } \\
\text { protein, ApbE* }\end{array}$ & 348 \\
\hline & $\begin{array}{l}\text { AMM82575. } \\
1\end{array}$ & $\begin{array}{l}\text { C4-dicarboxylate ABC } \\
\text { transporter substrate- } \\
\text { binding protein* }\end{array}$ & 339 \\
\hline
\end{tabular}

39

33

39

\section{H. ducreyi}

S. typhirium

P. multocida No protection serotype A

$\begin{array}{lll}13.53 & 6.88 & 55\end{array}$

$90.64 \quad 9.03 \quad 27$

$48.21 \quad 9.76$

$16.21 \quad 7.79$

$42.23 \quad 8.38$

$87.77 \quad 6.62$

$37.37 \quad 7.70$

$30.07 \quad 8.99$

$38.66 \quad 5.85$

$35.87 \quad 6.76$

$$
\text { V. }
$$

\section{Homologous}

No protection 


\begin{tabular}{|c|c|c|c|c|c|c|c|c|c|c|}
\hline $\begin{array}{l}\text { Secretion } \\
\text { system- }\end{array}$ & $\begin{array}{l}\text { AMM81343. } \\
1\end{array}$ & $\begin{array}{l}\text { TolC family protein, } \\
\text { TolC* }\end{array}$ & 455 & 50.71 & 9.14 & 26 & $\begin{array}{l}P . \quad \text { multocida } \\
\text { serotype A }\end{array}$ & No protection & $\begin{array}{l}\text { (Hatfaludi et } \\
\text { al., 2012) }\end{array}$ & Recombinant \\
\hline $\begin{array}{l}\text { efflux } \\
\text { pumps }\end{array}$ & $\begin{array}{l}\text { AMM82718. } \\
1\end{array}$ & $\begin{array}{l}\mathrm{Na}(+) \text {-translocating } \\
\mathrm{NADH} \text {-quinone reductase }\end{array}$ & 260 & 27.62 & 6.60 & 9 & $V$. alginolyticus & & $\begin{array}{l}\text { (Nakayama et } \\
\text { al. 2000) }\end{array}$ & \\
\hline proteins & & subunit $\mathrm{C}, \mathrm{NqrC}^{*}$ & & & & & $V$. cholerae & & $\begin{array}{l}\text { (Barquera et } \\
\text { al., 2001) }\end{array}$ & \\
\hline & & & & & & & $V$. barveyi & & $\begin{array}{l}\text { (Borshchevsk } \\
\text { iy et al., 2015) }\end{array}$ & \\
\hline \multirow[t]{4}{*}{$\begin{array}{l}\text { Metabolism } \\
\text {-related } \\
\text { proteins }\end{array}$} & $\begin{array}{l}\text { AMM82444. } \\
1\end{array}$ & $\begin{array}{l}\text { Glycerophosphodiester } \\
\text { phosphodiesterase, GlpQ* }\end{array}$ & 358 & 41.16 & 6.57 & 20 & $\begin{array}{l}\text { A. } \\
\text { pleuropneumoniae, } \\
\text { P. multocida, } \\
\text { H. inlfuenzae }\end{array}$ & & (Gouré et al., & \\
\hline & $\begin{array}{l}\text { AMM82047. } \\
1\end{array}$ & $\begin{array}{l}\text { 6-phosphofructokinase, } \\
\text { PfkA* }\end{array}$ & 188 & 21.92 & 9.55 & 9 & S. aureus & & $\begin{array}{l}\text { (Vitko et al., } \\
\text { 2015) }\end{array}$ & \\
\hline & $\begin{array}{l}\text { AMM82071. } \\
1\end{array}$ & $\begin{array}{l}\text { NADP-specific glutamate } \\
\text { dehydrogenase, GdhA }\end{array}$ & 449 & 48.31 & 6.19 & 19 & $\begin{array}{l}P . \quad \text { multocida } \\
\text { serotype } \mathrm{B}: 2\end{array}$ & Homologous & $\begin{array}{l}\text { (Rafidah et al., } \\
\text { 2012; Rafidah }\end{array}$ & $\begin{array}{l}\text { Live } \\
\text { attenuated }\end{array}$ \\
\hline & & & & & & & & & $\begin{array}{l}\& \quad \text { Zamri- } \\
\text { Saad 2013) }\end{array}$ & \\
\hline \multirow{8}{*}{$\begin{array}{l}\text { Adherence } \\
\text { and } \\
\text { invasion- } \\
\text { related } \\
\text { proteins }\end{array}$} & $\begin{array}{l}\text { AMM82057. } \\
1\end{array}$ & $\begin{array}{l}\text { YopT-type cysteine } \\
\text { protease domain- } \\
\text { containing protein }\end{array}$ & 2613 & 286.54 & 6.06 & 105 & $\begin{array}{l}P . \quad \text { multocida } \\
\text { serotype } \mathrm{A}, \mathrm{B}, \mathrm{D}\end{array}$ & & $\begin{array}{l}\text { (Shayegh et } \\
\text { al., 2010) }\end{array}$ & \\
\hline & $\begin{array}{l}\text { AMM82054. } \\
1\end{array}$ & $\begin{array}{l}\text { YopT-type cysteine } \\
\text { protease domain- } \\
\text { containing protein }\end{array}$ & 4096 & 449.84 & 5.69 & 168 & & & & \\
\hline & $\begin{array}{l}\text { AMM81494. } \\
1\end{array}$ & Host specificity protein J & 1215 & 136.12 & 5.04 & 54 & $\begin{array}{l}P . \quad \text { multocida } \\
\text { serotype } \mathrm{A}\end{array}$ & \multirow[t]{6}{*}{ No protection } & $\begin{array}{l}\text { (Hatfaludi et } \\
\text { al., 2012) }\end{array}$ & \multirow[t]{3}{*}{ Recombinant } \\
\hline & $\begin{array}{l}\text { AMM81374. } \\
1\end{array}$ & $\begin{array}{l}\text { Tape measure domain- } \\
\text { containing protein }\end{array}$ & 604 & 63.04 & 9.66 & 17 & $\begin{array}{l}P . \quad \text { multocida } \\
\text { serotype A }\end{array}$ & & $\begin{array}{l}\text { (Sahay et al., } \\
\text { 2018) }\end{array}$ & \\
\hline & $\begin{array}{l}\text { AMM81035. } \\
1\end{array}$ & $\begin{array}{l}\text { Autotransporter domain- } \\
\text { containing protein, } \mathrm{NanH}\end{array}$ & 1055 & 117.73 & 8.92 & 48 & V. cholerae & & $\begin{array}{l}\text { (Jermyn et al., } \\
\text { 2002) }\end{array}$ & \\
\hline & \multirow[t]{2}{*}{$\begin{array}{l}\text { AMM81010. } \\
1\end{array}$} & \multirow[t]{2}{*}{$\begin{array}{l}\text { Acetylglucosamine } \\
\text { transferase, Opa }\end{array}$} & \multirow[t]{2}{*}{197} & \multirow[t]{2}{*}{21.76} & \multirow[t]{2}{*}{9.34} & \multirow[t]{2}{*}{8} & N. meningitidis & & $\begin{array}{l}\text { (Callaghan et } \\
\text { al., 2011) }\end{array}$ & Recombinant \\
\hline & & & & & & & $\begin{array}{l}P . \quad \text { multocida } \\
\text { serotype } \mathrm{A}\end{array}$ & & $\begin{array}{l}\text { Johnson et } \\
\text { al., 2013; Yu } \\
\text { et al., 2016; } \\
\text { Peng et al., } \\
2017 \text { ) }\end{array}$ & \\
\hline & $\begin{array}{l}\text { AMM82292. } \\
1\end{array}$ & $\begin{array}{l}\text { Translocation/assembly } \\
\text { module, TamB }\end{array}$ & 1300 & 142.19 & 5.77 & 65 & B. burgdorferi & & $\begin{array}{l}\text { (Iqbal et al., } \\
\text { 2016) }\end{array}$ & \\
\hline
\end{tabular}




$$
\begin{array}{ll}
\text { AMM82058. } & \begin{array}{l}
\text { Hemolysin } \\
\text { secretion/activation } \\
\text { protein, FhaC }
\end{array}
\end{array}
$$

AMM82567. Surface protein, adhesin

AMM82908. Peptidase family M48*

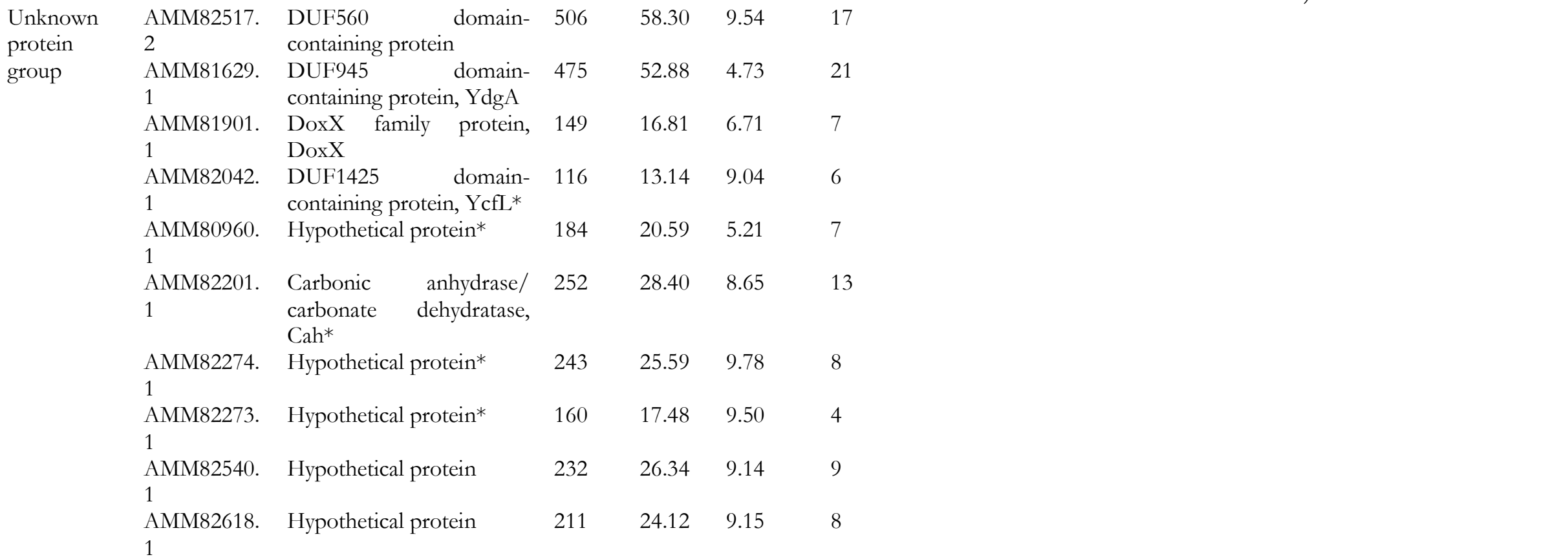

P. $\quad$ multoida No protection serotype A

A. baumannii

46

$\begin{array}{llll}256 & 27.96 & 9.45 & 15\end{array}$

M. tuberculosis

Homologous

E. coli

(Be08)

2008)

(Narita et al. al., 2012)

(Pérez et al.

2017)

\section{et al., DNA}

Narita

The proteins marked with asterisks are the determined lipoproteins. 
Several antigens were previously identified in $P$. multocida serotype B strains from India (Prasannavadhana et al., 2014) and China (Du et al., 2017) that were also acknowledged in this study, respectively. Based on Prasannavadhana et al. (2014) findings, similar proteins were included in this study as well, with $38.81 \%$ of the identified protein numbers. Meanwhile, based on Du et al. (2017) findings, six vaccine antigens were chosen, and three of the antigens were identified in this study. Both of these reports' findings pertained with proteomic analysis such as mass spectrometry in identifying the antigens from respective organisms. Nonetheless, the approaches used by these reports are required to follow on the verification of our determined antigens. In addition, some of the identified hypothetical antigens of other studies possibly are identified as the hypothetical antigens reported in this study. However, further functional analyses towards these proteins are needed to identify their role in pathogenicity and virulence. However, this study has enabled the identification of putative antigenic OMPs by analysing the genome to identify the antigens of the organism. Even though sophisticated tools were not applied, numerous OMPs were successfully determined. Besides, this study focuses on the Southeast Asian strain particularly isolated from Malaysia. Hence, certain OMPs are more likely to be unique to this strain.

Prediction of binding epitopes is crucial in the determination of vaccine design. Prediction by using pseudosequence similar HLA alleles was to observe the putative antigens that able to bind to bovine leukocyte antigens which are lacking by B cell epitope prediction. This is to ensure binding of the putative antigens towards the immune components of the host. Moreover, VaxiJen is commonly applied to complement epitopes identification (Zaharieva et al., 2017) and combining these tools, 53 putative antigens were determined. Nine putative antigenic OMPs were narrowed down as potential vaccine targets for HS. Two proteins from the porins group were known as major outer membrane proteins, OmpA and OmpH (Table 1). Both proteins determined in various $P$. multocida strains were strongly found to be immunogenic in various hosts (Tan et al., 2010; Gong et al., 2013; Joshi et al., 2013; Prasannavadhana et al. 2014; Du et al., 2017). Meanwhile OmpW and $\mathrm{OmpV}$ were reported as non-immunogenic in $P$. multocida but immunogenic in other pathogenic Gram-negative bacteria such as E. coli, S. flexneri, $V$. anguillarum and NTHi (Bernardini et al., 1993; Bolduc et al., 2000; McClean, 2012; Wu et al., 2013; Baliga et al., 2018;).

Interestingly, from this study, YopT protein was also identified as PfhA protein. The $p f h A$ gene was reported to be highly associated with bovine isolates in Iran and hence, it served as important pathogenic marker of bovine isolates (Shayegh et al., 2010). Opa or known as opacity associated protein, was identified as potential virulent associated element in three reported virulent $P$. multocida strains (P. multocida strain HB02, $P$. multocida strain X73 and P. multocida strain P1059) and also in this study (Table 1) but was never found in P. multocida strain Pm70 (Johnson et al., 2013; Yu et al., 2016; Peng et al., 2017). Immunization with recombinant Opa has enabled promotion of cross-reactive bactericidal antibodies (Callaghan et al., 2011). TamB that form TAM complex with TamA is known as putative antigen (Table 1). Deletion of the TAM complex has affected the colonization capabilities and virulence of several pathogenic bacteria such as S. enterica and $V$. fischeri (Heinz et al., 2015). This concluded the role of the complex as autotransporter translocation across the bacterial outer membrane particularly in the secretion of virulence associated OMPs (Iqbal et al., 2016). However, information on the pathogenicity of hypothetical proteins AMM82273.1 and AMM82618.1 were minimal due to insufficient comprehension towards the structure and function of these proteins. Confirmation of the immunogenicity of the selected putative antigenic OMPs is essential by using several analyses such as Western blot or ELISA. Furthermore, the suitability of vaccine design of each vaccine targets must be confirmed particularly for future live attenuated vaccine development.

Currently used bacterin vaccines have apparent limitations in HS prevention. De Alwis (1999) recommended live vaccine as an alternative to other types of vaccines due to the major advantage of being able to mimic natural route of infection leading to better and longer protection 
towards the animals in controlling disease outbreaks. Currently, there are two significant live vaccine candidates developed for HS prevention. A Sri Lankan strain of P. multocida B:2 (P. multocida B:2 85020) was attenuated with a deletion of a housekeeping gene, aro $A$, known as $P$. multocida B:2 JRMT12 (Tabatabaei et al., 2002). The live vaccine candidate was observed to immunize buffalo calves even with a high dosage of intramuscular (i.m.) vaccination and challenge without any disease symptoms shown (Hodgson et al., 2005).

Another HS live vaccine candidate known as P. multocida B:2 GDH7 was attenuated by the disruption of a housekeeping gene, $g d h A$, from a wild-type strain isolated from an outbreak case in Malaysia. The vaccine strain has allowed in promoting mass vaccination and prolonged protection in vaccinated animals (Rafidah et al., 2012, 2013). Protection and safety were analysed in respective studies to ensure the effectiveness of the vaccines to be used as a live vaccine for HS. This strain can also be further manipulated in order to generate a new mutant with each of the putative antigenic OMPs which might provide heterologous protection towards P. multocida and hence, increasing the vaccination coverage. Both potential vaccine strains were also reported to enable plasmid DNA delivery into the host cells (Othman et al., 2013; Kamal et al., 2017) making both strains a potential DNA vaccine delivery vectors. It is important that the manipulation of the OMPs in the attenuated bacterial strains will still allow the bacterial viability and safety in order to be used as a live vaccine.

In conclusion, through the application of three bioinformatics predictors, 105 putative OMPs were identified from the whole proteome of a Malaysian isolate, P. multocida subsp. multocida PMTB2.1. This study also enabled the determination of 53 putative antigenic OMPs from $P$. multocida serotype B:2 to be manipulated for future vaccine development against HS. The putative antigenic OMPs were focused by the antigenicity score filtering to only nine as potential vaccine candidates. Shortly, in-depth structural and functional evaluation of the selected antigens should be carried out together with the assessment of their pathogenesis and virulence. This will be an added value in the enhancement of the forthcoming HS vaccine development in order to ensure a safe and sustainable vaccination to be included in the bovine healthcare program.

\section{ACKNOWLEDGEMENTS}

FMA was supported by the academic grant awarded from SEARCA for UPM-KU Dual Degree program. FMA was also supported by the Graduate Research Fellowship awarded from School of Graduate Studies of UPM. This project was part of research funded under PRGS grant awarded (PRGS/1/2015/WAB01/UPM/01/1) by the Department of Higher Education, Ministry of Education Malaysia. This project was also supported by Universiti Putra Malaysia research grant (GP/2017/9560000).

\section{REFERENCES}

Al-Hasani, K., Boyce, J., McCarl, V. P., Bottomley, S., Wilkie, I., \& Adler, B. 2007. Identification of novel immunogens in Pasteurella multocida. Microbial Cell Factories 6(3).

Baliga, P., Shekar, M., \& Venugopal, M. N. 2018. Potential outer membrane protein candidates for vaccine development against the pathogen Vibrio anguillarum: A reverse vaccinology based identification. Current Microbiology 75(3): 368-377.

Barquera, B., Häse, C. C., \& Gennis, R. B. 2001. Expression and mutagenesis of the NqrC subunit of the NQR respiratory $\mathrm{Na}+$ pump from Vibrio cholerae with covalently attached FMN. FEBS Letters 492(1-2): 45-49.

Basagoudanavar, S. H., Singh, D. K., \& Varshney, B. C. 2006. Immunization with outer membrane proteins of Pasteurella multocida (6:B) provides protection in mice. Journal of Veterinary Medicine. A, Physiology, Pathology, Clinical Medicine 53(10): 524-530.

Benkirane, A., \& De Alwis, M. C. L. 2002. Haemorrhagic septicaemia, its significance, prevention and control in Asia. Veterinarni Medicina 47(8): 234-240.

Bernardini, M. L., Sanna, M. G., Fontaine, A., \& Sansonetti, P. J. 1993. OmpC is involved in invasion of epithelial cells by Shigella flexneri. Infection and Immunity 61(9): 3625-3635.

Berven, F. S., Flikka, K., Jensen, H. B., \& Eidhammer, I. 2004. BOMP: a program to predict integral beta-barrel outer membrane proteins encoded within genomes of Gramnegative bacteria. Nucleic Acids Research 32(Web Server issue): W394-9.

Bolduc, G. R., Bouchet, V., Jiang, R. Z., Geisselsoder, J., TruongBolduc, Q. C., Rice, P. A., Pelton, S. I., \& Goldstein, R. 2000. Variability of outer membrane protein P1 and its evaluation as a vaccine candidate against experimental otitis media due to nontypeable Haemophilus influenzae: An unambiguous, multifaceted approach. Infection and Immunity 68(8): 4505-4517.

Borshchevskiy, V., Round, E., Bertsova, Y., Polovinkin, V., Gushchin, I., Ishchenko, A., Kovalev, K., Mishin, A., Kachalova, G., Popov, A., Bogachev, A., \& Gordeliy, V. 
2015. Structural and functional investigation of flavin binding center of the $\mathrm{NgrC}$ subunit of sodium-translocating NADH: Quinone oxidoreductase from Vibrio harveyi. PLOS ONE 10(3): 1-13.

Bottero, D., Gaillard, M. E., Basile, L. A., Fritz, M., \& Hozbor, D. F. 2012. Genotypic and phenotypic characterization of Bordetella pertussis strains used in different vaccine formulations in Latin America. Journal of Applied Microbiology 112(6): 1266-1276.

Breau, C., Cameron, D. W., Desjardins, M., \& Lee, B. C. 2012. Oral immunization using $\mathrm{HgbA}$ in a recombinant chancroid vaccine delivered by attenuated Salmonella typhimurium SL3261 in the temperature-dependent rabbit model. Journal of Immunological Methods 375(1-2): 232-242.

Brun, P., Zumbo, A., Castagliuolo, I., Delogu, G., Manfrin, F., Sali, M., Fadda, G., Grillot-Courvalin, C., Palù, G., \& Manganelli, R. 2008. Intranasal delivery of DNA encoding antigens of Mycobacterium tuberculosis by non-pathogenic invasive Escherichia coli. Vaccine 26(16): 1934-1941.

Callaghan, M. J., Lewis, S., Sadarangani, M., Bailey, S. E. S., Chan, H., Ferguson, D. J. P., Derrick, J. P., Feavers, I., Maiden, M. C., \& Pollard, A. J. 2011. Potential of recombinant opa proteins as vaccine candidates against hyperinvasive meningococci. Infection and Immunity 79(7): 2810-2818.

Cao, P., Guo, D., Liu, J., Jiang, Q., Xu, Z., \& Qu, L. 2017. Genome-wide analyses reveal genes subject to positive selection in Pasteurella multocida. Frontiers in Microbiology 8(MAY):

Dabo, S. M., Confer, A., Montelongo, M., York, P., \& Wyckoff, J. H. 2008. Vaccination with Pasteurella multocida recombinant OmpA induces strong but non-protective and deleterious Th2-type immune response in mice. Vaccine 26(34): 43454351.

De Alwis, M. C. L. 1999. Haemorrhagic Septicaemia. ACLAR Monograph 57: 141.

Doytchinova, I. A., \& Flower, D. R. 2007. Vaxijen: a server for prediction of protective antigens, tumour antigens and subunit vaccines. BMC Bioinformatics 8(1): 4.

Du, H., Wu, C., Li, C., Fang, R., Ma, J., Ji, J., Li, Z., Li, N., Peng, Y., \& Zhou, Z. 2017. Two novel cross-protective antigens for bovine Pasteurella multocida. Molecular Medicine Reports 16(4): 4627-4633.

E-komon, T., Burchmore, R., Herzyk, P., \& Davies, R. 2012. Predicting the outer membrane proteome of Pasteurella multocida based on consensus prediction enhanced by results integration and manual confirmation. BMC Bioinformatics 13(1): 63.

Farrell, D., Jones, G., Pirson, C., Malone, K., Rue-Albrecht, K., Chubb, A. J., Vordermeier, M., \& Gordon, S. V. 2016. Integrated computational prediction and experimental validation identifies promiscuous $T$ cell epitopes in the proteome of Mycobacterium bovis. Microbial Genomics 2(8): 116.

Ferrer-Navarro, M., Ballesté-Delpierre, C., Vila, J., \& Fàbrega, A. 2016. Characterization of the outer membrane subproteome of the virulent strain Salmonella typhimurium SL1344. Journal of Proteomics 146 141-147.

Fusco, W. G., Afonina, G., Nepluev, I., Cholon, D. M., Choudhary, N., Routh, P. A., Almond, G. W., Orndorff, P. E., Staats, H., Hobbs, M. M., Leduc, I., \& Elkins, C. 2010. Immunization with the Haemophilus ducreyi hemoglobin receptor HgbA with adjuvant monophosphoryl lipid A protects swine from a homologous but not a heterologous challenge. Infection and Immunity 78(9): 3763-3772.

Garrido, M. E., Bosch, M., Bigas, A., Badiola, I., Barb., J., \& Llagostera, M. 2008. Heterologous protective immunization elicited in mice by Pasteurella multocida fur ompH. International Microbiology 11(1): 17-24.
Gatto, N. T., Dabo, S. M., Hancock, R. E., \& Confer, A. W. 2002. Characterization of, and immune responses of mice to, the purified OmpA-equivalent outer membrane protein of Pasteurella multocida serotype A:3 (Omp28). Veterinary Microbiology 87(3): 221-235.

Gong, Q., Qu, N., Niu, M., Qin, C., Cheng, M., Sun, X., \& Zhang, A. 2013. Immune responses and protective efficacy of a novel DNA vaccine encoding outer membrane protein of avian Pasteurella multocida. Veterinary Immunology and Immunopathology 152(3-4): 317-324.

Gouré, J., Findlay, W. A., Deslandes, V., Bouevitch, A., Foote, S. J., MacInnes, J. I., Coulton, J. W., Nash, J. H. E., \& Jacques, M. 2009. Microarray-based comparative genomic profiling of reference strains and selected Canadian field isolates of Actinobacillus pleuropneumoniae. BMC Genomics 10.

Hagan, C. L., Silhavy, T. J., \& Kahne, D. 2011. $\beta$-barrel membrane protein assembly by the Bam complex. Annual Review of Biochemistry 80(1): 189-210.

Hatfaludi, T., Al-Hasani, K., Gong, L., Boyce, J. D., Ford, M., Wilkie, I. W., Quinsey, N., Dunstone, M. A., Hoke, D. E., \& Adler, B. 2012. Screening of 71 P. multocida proteins for protective efficacy in a fowl cholera infection model and characterization of the protective antigen PlpE. PLoS ONE 7(7): 1-11.

Heinz, E., Selkrig, J., Belousoff, M. J., \& Lithgow, T. 2015. Evolution of the translocation and assembly module (TAM). Genome Biology and Evolution 7(6): 1628-1643.

Hodgson, J. C., Finucane, A., Dagleish, M. P., Ataei, S., Parton, R., \& Coote, J. G. 2005. Efficacy of vaccination of calves against hemorrhagic septicemia with a live aroA Derivative of Pasteurella multocida B: 2 by two different routes of administration efficacy of vaccination of calves against hemorrhagic septicemia with a live aroA derivative. Infection and Immunity 73(3): 1475-1481.

Iqbal, H., Kenedy, M. R., Lybecker, M., \& Akins, D. R. 2016. The TamB ortholog of Borrelia burgdorferi interacts with the $\beta$ barrel assembly machine (BAM) complex protein BamA. Molecular Microbiology 102(5): 757-774.

Jermyn, W. S., \& Boyd, E. F. 2002. Characterization of a novel Vibrio pathogenicity island (VPI-2) encoding neuraminidase (nanH) among toxigenic Vibrio cholerae isolates. In Microbiology (Vol. 148, Issue 11, pp. 3681-3693).

Johnson, T. J., Abrahante, J. E., Hunter, S. S., Hauglund, M., Tatum, F. M., Maheswaran, S. K., \& Briggs, R. E. 2013. Comparative genome analysis of an avirulent and two virulent strains of avian Pasteurella multocida reveals candidate genes involved in fitness and pathogenicity. BMC Microbiol 13(1): 106.

Joshi, S., Tewari, K., \& Singh, R. 2013. Comparative immunogenicity and protective efficacy of different preparations of outer membrane proteins of Pasteurella multocida (B:2) in a mouse model. Veterinarski Arbiv 83(6): 665-676.

Juncker, A., \& Willenbrock, H. 2003. Prediction of lipoprotein signal peptides in Gram negative bacteria. Protein Science 12(8): 1652-1662.

Kamal, N. M., Zamri-Saad, M., Masarudin, M. J., \& Othman, S. 2017. Interaction between Pasteurella multocida B:2 and its derivatives with bovine aortic endothelial cell (BAEC). $B M C$ Veterinary Research 13(1): 186.

Kolaskar, A. S., \& Tongaonkar, P. C. 1990. A semi-empirical method for prediction of antigenic determinants on protein antigens. FEBS Letters 276(1-2): 172-174.

Kumar, A., Mohanty, N. N., Chacko, N., Yogisharadhya, R., \& Shivachandra, S. B. 2014. Structural features of a highly conserved Omp16 protein of Pasteurella multocida strains and comparison with related peptidoglycan-associated lipoproteins (PAL). Indian Journal of Microbiology 55(1): 50 
56.

Lee, J., Kim, Y. B., \& Kwon, M. 2007. Outer membrane protein H for protective immunity against Pasteurella multocida. Journal of Microbiology (Seoul, Korea).

Liu, R., Zhang, P., Su, Y., Lin, H., Zhang, H., Yu, L., Ma, Z., \& Fan, H. 2016. A novel suicide shuttle plasmid for Streptococcus suis serotype 2 and Streptococcus equi ssp. rooepidemicus gene mutation. Scientific Reports 6.

Liu, Y. F., Yan, J. J., Lei, H. Y., Teng, C. H., Wang, M. C., Tseng, C. C., \& Wu, J. J. 2012. Loss of outer membrane protein C in Escherichia coli contributes to both antibiotic resistance and escaping antibody-dependent bactericidal activity. Infection and Immunity 80(5): 1815-1822.

Lodish, H., Berk, A., Kaiser, C. A., Kreiger, M., Scott, M. P., Bretscher, A., Ploegh, H., \& Matsudaira, P. 2007. Molecular Cell Biology (6th ed.). W. H. Freeman and Company.

Luo, Y., Zeng, Q., Glisson, J. R., Jackwood, M. W., Cheng, I. H. N., \& Wang, C. 1999. Sequence analysis of Pasteurella multocida major outer membrane protein $(\mathrm{OmpH})$ and application of synthetic peptides in vaccination of chickens against homologous strain challenge. Vaccine 17(7-8): 821831.

Maione, D., Margarit, I., Rinaudo, C. D., Masignani, V., Scarselli, M., Tettelin, H., Brettoni, C., Iacobini, E. T., Agostino, N. D., Miorin, L., Buccato, S., Mariani, M., Nogarotto, R., Dei, V. N., Vegni, F., Fraser, C., Teti, G., Madoff, L. C., Paoletti, L. C., ... Grandi, G. 2005. Identification of a universal group B Streptococcus vaccine by multiple genome screen. Science 309(5731): 148-150.

Mao, Z., Yu, L., You, Z., Wei, Y., \& Liu, Y. 2007. Cloning, expression and immunogenicty analysis of five outer membrane proteins of Vibrio parahaemolyticus zi2003. Fish and Shellfish Immunology 23(3): 567-575.

McClean, S. 2012. Eight stranded \& beta; -barrel and related outer membrane proteins: Role in bacterial pathogenesis. Protein \& Peptide Letters 19(10): 1013-1025.

Mohd Aris, A., Md Yasin, I.-S., Zamri-Saad, M., Mohd Daud, H., \& Mohamed Alipiah, N. 2016. Molecular characterization of Vibrio harveyi virulence-associated serine protease and outer membrane protein genes for vaccine development. International Journal of Biosciences (IJB) 8(3): 10-28.

Nakayama, Y., Yasui, M., Sugahara, K., Hayashi, M., \& Unemoto, T. 2000. Covalently bound flavin in the NqrB and NqrC subunits of $\mathrm{Na}(+)$-translocating $\mathrm{NADH}$-quinone reductase from Vibrio alginolyticus. FEBS Letters 474(2-3): 165-168.

Narita, S. I., Masui, C., Suzuki, T., Dohmae, N., \& Akiyama, Y. 2013. Protease homolog BepA (YfgC) promotes assembly and degradation of -barrel membrane proteins in Escherichia coli. Proceedings of the National Academy of Sciences 110(38): E3612-E3621.

Othman, S., Roe, A. J., Parton, R., \& Coote, J. G. 2013. Use of a dual reporter plasmid to demonstrate Bactofection with an attenuated AroA(-) derivative of Pasteurella multocida B:2. PloS One 8(8): e71524.

Pandey, N., Monendra, G., \& Rai, A. 2016. Conservation of properties of outer membranes protein across host genera of Pasteurella multocida suggests common mechanism of action. Molecular Biology 5(2).

Pati, U. S., Srivastava, S. K., Roy, S. C., \& More, T. 1996. Innmunogenicity of outer membrane protein of Pasteurella multocida in buffalo calves. Veterinary Microbiology 52 301311.

Peng, Z., Liang, W., Liu, W., Chen, H., \& Wu, B. 2017. Genome characterization of Pasteurella multocida subspecies septica and comparison with Pasteurella multocida subspecies multocida and gallicida. Archives of Microbiology 199(4): 635640.

Pérez, A., Merino, M., Rumbo-Feal, S., Álvarez-Fraga, L., Vallejo,
J. A., Beceiro, A., Ohneck, E. J., Mateos, J., FernándezPuente, P., Actis, L. A., Poza, M., \& Bou, G. 2017. The $\mathrm{FhaB} / \mathrm{FhaC}$ two-partner secretion system is involved in adhesion of Acinetobacter baumannii AbH12o-A2 strain. Virulence 8(6): 959-974.

Poppleton, D. I., Duchateau, M., Hourdel, V., Matondo, M., Flechsler, J., Klingl, A., Beloin, C., \& Gribaldo, S. 2017. Outer membrane proteome of Veillonella parvula: A diderm firmicute of the human microbiome. Frontiers in Microbiology 8(JUN): 1215.

Prasannavadhana, A., Kumar, S., Thomas, P., Sarangi, L. N., Gupta, S. K., Priyadarshini, A., Nagaleekar, V. K., \& Singh, V. P. 2014. Outer membrane proteome analysis of Indian strain of Pasteurella multocida serotype B:2 by MALDITOF/MS analysis. Scientific World Journal 2014.

Rafidah, O., \& Zamri-Saad, M. 2013. Effect of dexamethasone on protective efficacy of live gdhA derivative Pasteurella multocida B:2 vaccine. Asian Journal of Animal and Veterinary Advances 8(3): 548-554.

Rafidah, O., Zamri-Saad, M., Shahirudin, S., \& Nasip, E. 2012. Efficacy of intranasal vaccination of field buffaloes against haemorrhagic septicaemia with a live gdhA derivative Pasteurella multocida B:2. Veterinary Record 171(7): 175.

Rappuoli, R. 2001. Reverse vaccinology, a genome-based approach to vaccine development. Vaccine 19(17-19): 2688-2691.

Rigel, N. W., Schwalm, J., Ricci, D. P., \& Silhavy, T. J. 2012. BamE modulates the Escherichia coli beta-barrel assembly machine component BamA. Journal of Bacteriology 194(5): 1002-1008.

Sahay, S., Shome, R., Sankarasubramanian, J., Vishnu, U. S., Prajapati, A., Natesan, K., Shome, B. R., Rahman, H., \& Rajendhran, J. 2018. Insights into the genome sequence of ovine Pasteurella multocida type A strain associated with pneumonic pasteurellosis. Small Ruminant Research.

Santana-Jorge, K. T. O., Santos, T. M., Tartaglia, N. R., Aguiar, E. L., Souza, R. F. S., Mariutti, R. B., Eberle, R. J., Arni, R. K., Portela, R. W., Meyer, R., \& Azevedo, V. 2016. Putative virulence factors of Corynebacterium psendotuberculosis FRC41: Vaccine potential and protein expression. Microbial Cell Factories 15(1): 83.

Shahin, K., Thompson, K. D., Inglis, N. F., Mclean, K., RamirezParedes, J. G., Monaghan, S. J., Hoare, R., Fontaine, M., Metselaar, M., \& Adams, A. 2018. Characterization of the outer membrane proteome of Francisella noatunensis subsp. orientalis. Journal of Applied Microbiology 125(3): 686-699.

Shavegh, J., Atashpaz, S., Salehi, T. Z., \& HEJAZI, M. S. 2010. Potential of Pasteurella multocida isolated from healthy and diseased cattle and buffaloes in induction of diseases. Bulletin-Veterinary Institute in Pulany 54(3): 299-304.

Singh, R., Capalash, N., \& Sharma, P. 2017. Immunoprotective potential of BamA, the outer membrane protein assembly factor, against MDR Acinetobacter baumannii. Scientific Reports $7(1)$.

Srivastava, S. K. 1998. Outer membrane protein of Pasteurella multocida serotype B:2 is immunogenic and antiphagocytic. Indian Journal of Experimental Biology 36(5): 530-532.

Tabatabaei, M., Liu, Z., Finucane, A., Parton, R., \& Coote, J. 2002. Protective immunity conferred by attenuated aroA derivatives of Pasteurella multocida B:2 strains in a mouse model. Infection and Immunity 70(7): 3355-3362.

Tan, H. Y., Nagoor, N. H., \& Sekaran, S. D. 2010. Cloning, expression and protective capacity of $37 \mathrm{kDa}$ outer membrane protein gene $(\mathrm{ompH})$ of Pasteurella multocida serotype B:2. Tropical Biomedicine 27(3): 430-441.

Thorpe, C., Edwards, L., Snelgrove, R., Finco, O., Rae, A., Grandi, G., Guilio, R., \& Hussell, T. 2007. Discovery of a vaccine antigen that protects mice from Chlamydia pneumoniae infection. Vaccine 25(12): 2252-2260.

Tidhar, A., Flashner, Y., Cohen, S., Levi, Y., Zauberman, A., Gur, 
D., Aftalion, M., Elhanany, E., Zvi, A., Shafferman, A., \& Mamroud, E. 2009. The NIpD lipoprotein is a novel Yersinia pestis virulence factor essential for the development of plague. PLoS ONE 4(9): 7023.

Vitko, N. P., Spahich, N. A., \& Richardson, A. R. 2015. Glycolytic dependency of high-level nitric oxide resistance and virulence in Staphylococcus aureus. MBio 6(2).

Willis, L. M., Stupak, J., Richards, M. R., Lowary, T. L., Li, J., \& Whitfield, C. 2013. Conserved glycolipid termini in capsular polysaccharides synthesized by ATP-binding cassette transporter-dependent pathways in Gram-negative pathogens. Proceedings of the National Academy of Sciences 110(19): 7868-7873.

Wu, X. Bin, Tian, L. H., Zou, H. J., Wang, C. Y., Yu, Z. Q., Tang, C. H., Zhao, F. K., \& Pan, J. Y. 2013. Outer membrane protein OmpW of Escherichia coli is required for resistance to phagocytosis. Research in Microbiology 164(8): 848-855.

Wu, J. R., Shien, J. H., Shieh, H. K., Chen, C. F., \& Chang, P. C. 2007. Protective immunity conferred by recombinant Pasteurella multocida lipoprotein E (PlpE). Vaccine 25(21): 4140-4148.

Yu, C., Sizhu, S., Luo, Q., Xu, X., Fu, L., \& Zhang, A. 2016. Genome sequencing of a virulent avian Pasteurella multocida strain GX-Pm reveals the candidate genes involved in the pathogenesis. Research in Veterinary Science 105 23-27.

Yu, N. Y., Wagner, J. R., Laird, M. R., Melli, G., Rey, S., Lo, R., Dao, P., Cenk Sahinalp, S., Ester, M., Foster, L. J., \& Brinkman, F. S. L. 2010. PSORTb 3.0: Improved protein subcellular localization prediction with refined localization subcategories and predictive capabilities for all prokaryotes. Bioinformatics 26(13): 1608-1615.

Zaharieva, N., Dimitrov, I., Flower, D. R., \& Doytchinova, I. 2017. Immunogenicity prediction by VaxiJen: $A$ ten year overview. Journal of Proteomics \& Bioinformatics 10(11): 298310.

Zamri-Saad, M. 2013. Haemorrbagic septicaemia of cattle \& buffaloes in Asia. Universiti Putra Malaysia Press. Retrieved from http://psasir.upm.edu.my/id/eprint/39200/

Zha, Z., Li, C., Li, W., Ye, Z., \& Pan, J. 2016. LptD is a promising vaccine antigen and potential immunotherapeutic target for protection against Vibrio species infection. Scientific Reports 6.

Zhang, G. L., Deluca, D. S., Keskin, D. B., Chitkushev, L., Zlateva, T., Lund, O., Reinherz, E. L., \& Brusic, V. 2011. MULTIPRED2: A computational system for large-scale identification of peptides predicted to bind to HLA supertypes and alleles. Journal of Immunology Methods 374(12): 53-61. 